\title{
An $8 \times 8$ Quasi-Orthogonal STBC Form for Transmissions over Eight or Four Antennas
}

\author{
Rohan Grover, Student Member, IEEE, Weifeng Su, Member, IEEE, and Dimitris A. Pados, Member, IEEE
}

\begin{abstract}
An $8 \times 8$ two-symbol decodable quasi-orthogonal space-time block code (QO-STBC) is presented which can be transmitted across either 8 or 4 antennas with full rate and the same full diversity order. For the 8-transmit-antenna system, a new expression is developed to identify rotation angles that maximize the diversity (eigenvalue) product. In addition, it is shown that the previously proposed sum-eigenvalue maximization criterion for the design of rotation angles is not relevant/applicable and an alternative minimum eigenvalue maximization criterion is suggested. Finally, new optimal rotation angles are obtained by working directly with a pairwise-error-probability (PEP) upperbound expression. For 4 -transmit-antenna systems and correlated channel fading conditions, the PEP-upper-bound is modified accordingly to take into account the channel correlation. Using the new PEP-upper-bound we obtain rotation angles that maximize the diversity product and find, contrary to previous results, that the optimized angles are independent of the correlation coefficient. Simulation studies initiated herein demonstrate the advantage of using the proposed codeword across 4 transmit antennas when compared with other $4 \times 4$ QO-STBC transmission schemes. For 8 transmit antennas, the studies compare the three selected rotation angle optimization criteria (diversity product, minimum eigenvalue, PEP-upper-bound).
\end{abstract}

Index Terms-Constellation rotation, diversity product, MIMO systems, pairwise-error-probability (PEP), quasi-orthogonal space-time block codes (QO-STBC), sum or minimum eigenvalue maximization.

\section{INTRODUCTION}

$\mathbf{O}$ RTHOGONAL space-time block codes (O-STBC) [1][3] achieve full transmit diversity and allow singlecomplex-symbol (or equivalently two real symbols) maximum likelihood (ML) decoding. The drawback of O-STBCs is that full-rate codewords do not exist for more than two transmit antennas. For the case of four transmit antennas, the rate limitation of O-STBCs was overcome by quasi-orthogonal (QO) STBCs at the expense of diversity loss [4]-[6]. Fullrate full-diversity quasi-orthogonal codewords for 4-transmitantennas were then presented in [7]-[9] by retaining the code

Manuscript received July 18, 2007; revised January 18, 2008; accepted March 29, 2008. The associate editor coordinating the review of this paper and approving it for publication is K. B. Lee.

This work was supported in part by the National Science Foundation under Grant CCF-0219903, the U.S. Air Force Office of Scientific Research under Grant FA9550-04-1-0256, and the U.S. Air Force Research Laboratory under Grant FA8750-08-1-0063. The U.S. Government is authorized to reproduce and distribute reprints for governmental purposes notwithstanding any copyright notation thereon. A part of this work was presented at IEEE Intern. Conf. on Acoustic Speech and Signal Proc. (ICASSP), Honolulu, Hawaii, April 2007.

R. Grover was with the Department of Electrical Engineering, State University of New York at Buffalo, Buffalo, NY 14260 USA. He is now with Radiospire Networks, Hudson, MA 01749 USA (e-mail: rgrover@radiospire.com).

W. Su and D. A. Pados are with the Department of Electrical Engineering, State University of New York at Buffalo, Buffalo, NY 14260 USA (e-mail: \{weifeng, pados\}@eng.buffalo.edu).

Digital Object Identifier 10.1109/T-WC.2008.070791 structure of [4], [5] and modifying the constellation of some of the symbols. ML decoding of the QO-STBCs in [7]-[9] requires joint detection of two complex symbols (or equivalently four real symbols). Interleaving real and imaginary parts of different symbols enables single-complex-symbol decoding of full-rank, full-diversity QO-STBCs for the 4-transmit-antenna case [10], [11], at the expense of some performance loss in comparison with joint two-symbol detection [7].

The codewords in [1]-[11] partition the symbols into orthogonal sets and ML detection requires only joint decoding of the symbols in each orthogonal set individually. Since the complexity of the ML decoder increases exponentially with the number of symbols in each orthogonal set, a trade-off between rate/diversity and decoding requirements is taking shape, especially for large number of transmit antennas. In [12], QOSTBCs for 8 transmit antennas that attain full diversity and full rate were presented that require, however, joint detection of four complex symbols (or equivalently eight real symbols). Reduction in complexity was achieved for the 8-antenna case through the process of interleaving the real and imaginary parts of different symbols [13], [14]; the codewords can be partitioned into four orthogonal sets and hence require joint two-complex-symbol decoding only. In [15], [16], codes that partition the symbols into four semi-orthogonal groups are proposed.

In this paper we present an alternative two-symboldecodable, full rate $8 \times 8$ QO-STBC form that can be applied across either 8-transmit or 4-transmit-antenna systems with the same full diversity order (making the 4-antenna option most appealing). The proposed QO-STBC employs constellation rotation (CR) and symbol-interleaving similar to [13], [14], however, the $8 \times 8$ codeword can be divided into two $4 \times 4$ codewords. We initially concentrate our efforts on the codeword for the 8-transmit-antenna system and evaluate conditions on the rotation angles necessary for the codeword to achieve full diversity order. Since several rotation angle pairs may exist that maximize the diversity order, we investigate different criteria for rotation angle selection to further improve error-rate performance. Common choice for rotation angle optimization of QO-STBCs is the maximization of the diversity product which in turn leads to minimization of the pairwiseerror-probability (PEP)-upper-bound at (asymptotically) high signal-to-noise ratios (SNR) [17]. However, for space-time codes with large diversity order and/or large number of transmit antennas, diversity product maximization may not provide satisfactory PEP-bound minimization and error-rate performance over operable SNRs [18]. Contrary to the popular choice of diversity product maximization, we pursue other means of optimizing the rotation angles to improve system performance. Within the class of two-symbol decodable, full 
rate $8 \times 8$ QO-STBCs we examine four different rotation angle optimization criteria: (i) We find a new expression for the rotation angles that maximize the diversity product of the suggested codeword. (ii) We show that sum-eigenvalue maximization as proposed in [18] is irrelevant/non-applicable to the 8-transmit-antenna QO-STBCs. (iii) We suggest, instead, and solve minimum-eigenvalue maximization. (iv) Finally, we use directly the PEP-upper-bound to obtain new true PEP-upperbound optimal rotation angles.

The rest of the paper deals with codeword design and rotation angle optimization for the 4-transmit-antenna system. For the 4-transmit-antenna system we allow the channel coefficients of the first $4 \times 4$ codeword block to be correlated with the channel coefficients of the next $4 \times 4$ codeword block. We show that as long as the correlation is less than $100 \%$, a diversity order of 8 is achieved using only 4 antennas. Using the PEPupper-bound results for fast fading time-correlated channels in [19], we re-evaluate the PEP-upper-bound to incorporate the channel correlation. We then optimize the rotation angles using the four criteria discussed above for the 8-transmitantenna case. We find that the rotation angles that maximize the diversity product for the uncorrelated 8-transmit-antenna system also maximize the diversity product for the correlated 4-transmit-antenna system. Arguably, this may be somewhat surprising because it did not seem possible in the past to make diversity product rotation angle optimization independent of the correlation coefficient [19]. For the other three criteria, we show that sum-eigenvalue maximization as proposed in [18] is again irrelevant/non-applicable to the 4-transmitantenna case, while the remaining two criteria (minimumeigenvalue minimization and PEP-upper-bound minimization) yield complicated, rather intractable optimization equations as they become dependent on the correlation coefficient.

The paper is organized as follows. In Section II we present the $(8 \times 8)$ code structure for 8 and 4 -transmit-antenna systems and describe pertinent ML decoding details. In Section III we examine the diversity order of the codeword for 8 transmit antennas; rotation-angle design criteria are analyzed in Section IV. Section V deals with codeword transmission, diversity order, and rotation angle optimization for the 4-transmitantenna case. Section VI presents simulation results in support of our theoretical developments. A few concluding remarks are drawn in Section VII.

\section{Code Structure And Transceiver Model}

Let $N_{t}$ be the number of transmit antennas, $N_{r}$ the number of receive antennas, and $T$ the number of time slots over which the code is transmitted. We denote the number of transmitted symbols by $K$. The eight symbols $a_{k}, k=$ $1, \ldots, K=8$, to be transmitted are formed by mapping the incoming bits onto known constellations, e.g. quadratureamplitude-modulated (QAM), while their corresponding constellation rotated version $\bar{a}_{k}, k=1, \ldots, K=8$, is created by

$$
\begin{aligned}
\bar{a}_{m} & =\left(a_{m R}+i a_{m I}\right) e^{i \phi}, m=1,2,5,6, \\
\bar{a}_{n} & =\left(a_{n R}+i a_{n I}\right) e^{i \theta}, \quad n=3,4,7,8,
\end{aligned}
$$

where $a_{k R}$ and $a_{k I}$ denote the real and imaginary part of the symbol $a_{k}$, respectively, and $\phi, \theta$, are the rotation angles to be optimized. The symbols $\bar{a}_{k}$ are interleaved to form $x_{k}, k=$ $1, \ldots, K=8$,

$$
\begin{array}{ll}
x_{1}=\bar{a}_{1 R}+i \bar{a}_{5 I}, & x_{2}=\bar{a}_{2 R}+i \bar{a}_{6 I}, \\
x_{3}=\bar{a}_{3 R}+i \bar{a}_{7 I}, & x_{4}=\bar{a}_{4 R}+i \bar{a}_{8 I}, \\
x_{5}=\bar{a}_{5 R}+i \bar{a}_{1 I}, & x_{6}=\bar{a}_{6 R}+i \bar{a}_{2 I}, \\
x_{7}=\bar{a}_{7 R}+i \bar{a}_{3 I}, & x_{8}=\bar{a}_{8 R}+i \bar{a}_{4 I} .
\end{array}
$$

We form/define two symbol matrices $\mathbf{X}_{1}$ and $\mathbf{X}_{2}$ as shown below

$$
\begin{aligned}
\mathbf{X}_{1} & =\left[\begin{array}{cccc}
x_{1} & x_{2} & x_{3} & x_{4} \\
-x_{2}^{*} & x_{1}^{*} & -x_{4}^{*} & x_{3}^{*} \\
x_{3} & x_{4} & x_{1} & x_{2} \\
-x_{4}^{*} & x_{3}^{*} & -x_{2}^{*} & x_{1}^{*}
\end{array}\right], \\
\mathbf{X}_{2} & =\left[\begin{array}{cccc}
x_{5} & x_{6} & x_{7} & x_{8} \\
-x_{6}^{*} & x_{5}^{*} & -x_{8}^{*} & x_{7}^{*} \\
x_{7} & x_{8} & x_{5} & x_{6} \\
-x_{8}^{*} & x_{7}^{*} & -x_{6}^{*} & x_{5}^{*}
\end{array}\right] .
\end{aligned}
$$

Consider now availability of either $N_{t}=8$ or $N_{t}=4$ transmit antennas. Below, we describe our transceiver model for each case.

Case of eight transmit antennas: We suggest the codeword

$$
\mathbf{X}=\left[\begin{array}{cc}
\mathbf{X}_{1} & \mathbf{0}_{\mathbf{4} \times \mathbf{4}} \\
\mathbf{0}_{\mathbf{4} \times \mathbf{4}} & \mathbf{X}_{2}
\end{array}\right]
$$

The $(T=8) \times N_{r}$ received signal matrix $\mathbf{Y}$ is given by

$$
\mathbf{Y}=\sqrt{\frac{\rho^{2} A}{N_{t}}} \mathbf{X H}+\mathbf{N}
$$

where $\rho^{2} A$ is the received signal energy at each receive antenna, $\mathbf{H}$ is the $8 \times N_{r}$ channel matrix and $\mathbf{N}$ is the $8 \times N_{r}$ noise matrix. The elements of $\mathbf{H}$ and $\mathbf{N}$ are modeled as independent and identically distributed complex Gaussian random variables of zero mean and unit variance without loss of generality; $\rho=\sqrt{2}$ satisfies the energy constraint $E\left\{\|\mathbf{X}\|_{F}^{2}\right\}=T N_{t}\left(E\{\cdot\}\right.$ is the expectation operator and $\|\cdot\|_{F}^{2}$ denotes Frobenius norm of a matrix). From an implementation point of view, reduction of the large peak-to-average-power ratio (PAPR) created by the zeros during transmission of (4) can be achieved by multiplying $\mathbf{X}$ by an $8 \times 8$ normalized Hadamard matrix before transmission. This effect is reversed at the receiver by multiplying the received signal matrix by the transpose of the Hadamard matrix. The net transceiver model still maintains the form in (4) and (5) by virtue of the orthogonality of the Hadamard matrix.

Case of four transmit antennas: Assume now that we have available only $N_{t}=4$ antennas. We transmit $\mathbf{X}_{1}$ in the first four time slots and $\mathbf{X}_{2}$ in the next four time slots. The $4 \times N_{r}$ received signal matrices $\mathbf{Y}_{1}$ and $\mathbf{Y}_{2}$ are given by

$$
\mathbf{Y}_{1}=\sqrt{\rho^{2} \frac{A}{N_{t}}} \mathbf{X}_{1} \mathbf{H}_{1}+\mathbf{N}_{1}, \mathbf{Y}_{2}=\sqrt{\rho^{2} \frac{A}{N_{t}}} \mathbf{X}_{2} \mathbf{H}_{2}+\mathbf{N}_{2} .
$$

Similar to the 8-transmit-antenna case, the elements of the $4 \times N_{r}$ matrices, $\mathbf{H}_{1}, \mathbf{H}_{2}, \mathbf{N}_{1}$ and $\mathbf{N}_{2}$ are modeled as identically distributed complex Gaussian random variables (with zero mean and unit variance); $\rho^{2} A$ is the received signal energy at each receive antenna; $\rho=1$ satisfies the energy 
constraint $E\left\{\left\|\mathbf{X}_{1}\right\|_{F}^{2}\right\}=E\left\{\left\|\mathbf{X}_{2}\right\|_{F}^{2}\right\}=T N_{t}$. Individually within a matrix, the entries are taken to be independent from each other; $\mathbf{N}_{1}$ as a whole is independent of $\mathbf{N}_{2}, \mathbf{H}_{1}$ and $\mathbf{H}_{2}$ are independent from $\mathbf{N}_{1}$ and $\mathbf{N}_{2}$, but $\mathbf{H}_{1}$ and $\mathbf{H}_{2}$ are characterized as potentially correlated with each other via a correlation coefficient $p$. To express the correlation between the elements of the two channel matrices we define the extended channel matrix $\mathbf{H}_{e} \triangleq\left[\begin{array}{ll}\mathbf{H}_{1}^{T} & \mathbf{H}_{2}^{T}\end{array}\right]^{T}$ and obtain

$E\left\{\mathbf{h}_{e j} \mathbf{h}_{e l}^{H}\right\}=\left\{\begin{array}{cc}{\left[\begin{array}{ll}\mathbf{I}_{4} & p \mathbf{I}_{4} \\ p \mathbf{I}_{4} & \mathbf{I}_{4}\end{array}\right], \quad j=l, \quad 1 \leq j, l \leq N_{r}} \\ \mathbf{0}_{8}, \quad \text { otherwise, }\end{array}\right.$

where $\mathbf{h}_{e j}, j=1, \ldots, N_{r}$, are the columns of $\mathbf{H}_{e}$ and $p$ is the correlation coefficient. In similar fashion to $\mathbf{H}_{e}$, the extended noise matrix may be defined as $\mathbf{N}_{e} \triangleq\left[\begin{array}{l}\mathbf{N}_{1}^{T} \mathbf{N}_{2}^{T}\end{array}\right]^{T}$. Combining $\mathbf{Y}_{1}$ and $\mathbf{Y}_{2}$ in (6) into a single equation we have

$$
\mathbf{Y}_{e}=\rho \sqrt{\frac{A}{N_{t}}} \mathbf{X H}_{e}+\mathbf{N}_{e}
$$

where $\mathbf{Y}_{e}=\left[\begin{array}{ll}\mathbf{Y}_{1}^{T} & \mathbf{Y}_{2}^{T}\end{array}\right]^{T}$ and $\mathbf{X}$ is defined in (4). It is important to note that (6) represents a system with timecorrelated channel coefficients while (8) represents a system in which the channel coefficients are correlated across space (channel coefficients of the four actual antennas correlated with the channel coefficients of the four virtual antennas). This distinction is important when obtaining the PEP-upper-bound for the 4-transmit-antenna system later in the presentation.

We now proceed with the description of the ML detector for both cases. Let $\mathcal{A}$ represent the symbol constellation and $\mathcal{Z}$ denote the set of $|\mathcal{A}|^{K}$ symbol vector points $(|\cdot|$ denotes cardinality of a set) in the complex $K$-dimensional space. If $f(\cdot)$ represents the one-to-one mapping of the symbol vector $\mathbf{a} \in \mathcal{A}^{K}$ into $\mathbf{X}$, for the 8-antenna case the ML estimate of the symbol vector assuming perfect channel state information at the receiver is

$$
\begin{aligned}
& \mathbf{a}_{M L}=\underset{\hat{\mathbf{a}} \in \mathcal{A}^{K}, \hat{\mathbf{X}}=f(\hat{\mathbf{a}})}{\operatorname{argmin}}\left\|\mathbf{Y}-\rho \sqrt{\frac{A}{N_{t}}} \hat{\mathbf{X}} \mathbf{H}\right\|_{F}^{2} \\
&=\underset{\hat{\mathbf{a}} \in \mathcal{A}^{K}, \hat{\mathbf{X}}=f(\hat{\mathbf{a}})}{\operatorname{argmin}} \sum_{j=1}^{N_{r}}\left\{\frac{A \rho^{2}}{N_{t}} \mathbf{h}_{j}^{H} \hat{\mathbf{X}}^{H} \hat{\mathbf{X}} \mathbf{h}_{j}\right. \\
&-\left.-2 \rho \sqrt{\frac{A}{N_{t}}} \operatorname{Re}\left\{\mathbf{y}_{j}^{H} \hat{\mathbf{X}} \mathbf{h}_{j}\right\}\right\}
\end{aligned}
$$

where $\mathbf{y}_{j}, \mathbf{h}_{j}, j=1, \ldots, N_{r}$, are the columns of $\mathbf{Y}$ and $\mathbf{H}$ respectively. Expanding $\hat{\mathbf{X}}^{H} \hat{\mathbf{X}}$ we have

$$
\hat{\mathbf{X}}^{H} \hat{\mathbf{X}}=\left[\begin{array}{cccc}
a \mathbf{I}_{2} & b \mathbf{I}_{2} & \mathbf{0} \\
b \mathbf{I}_{2} & a \mathbf{I}_{2} & \\
0 & c \mathbf{I}_{2} & d \mathbf{I}_{2} \\
\mathbf{0} & d \mathbf{I}_{2} & c \mathbf{I}_{2}
\end{array}\right]
$$

where

$$
\begin{array}{rcc}
a & \triangleq & \sum_{k=1}^{K}\left|\hat{x}_{k}\right|^{2}, \\
b & \triangleq & \sum_{k=1}^{K / 2} 2 \operatorname{Re}\left\{\hat{x}_{k}^{*} \hat{x}_{k+K / 2}\right\}, \\
c & \triangleq & \sum_{k=K+1}^{2 K}\left|\hat{x}_{k}\right|^{2}, \\
d & \triangleq & \sum_{k=K+1}^{K+K / 2} 2 \operatorname{Re}\left\{\hat{x}_{k}^{*} \hat{x}_{k+K / 2}\right\}, \quad K=4 .
\end{array}
$$

Substituting (1) and (2) in (11) we obtain

$$
\begin{array}{rlrl}
a & = & \hat{\bar{a}}_{1 R}^{2}+\hat{\bar{a}}_{2 R}^{2}+\hat{\bar{a}}_{3 R}^{2}+\hat{\bar{a}}_{4 R}^{2}+\hat{\bar{a}}_{5 I}^{2}+\hat{\bar{a}}_{6 I}^{2}+\hat{\bar{a}}_{7 I}^{2}+\hat{\bar{a}}_{8 I}^{2}, \\
b= & 2 \hat{\bar{a}}_{1 R} \overline{\bar{a}}_{3 R}+2 \hat{\bar{a}}_{2 R} \overline{\bar{a}}_{4 R}+2 \hat{\bar{a}}_{5 I} \hat{\bar{a}}_{7 I}+2 \overline{\bar{a}}_{6 I} \hat{\bar{a}}_{8 I}, \\
c & = & \hat{\bar{a}}_{5 R}^{2}+\hat{\bar{a}}_{6 R}^{2}+\hat{\bar{a}}_{7 R}^{2}+\hat{\bar{a}}_{8 R}^{2}+\hat{\bar{a}}_{1 I}^{2}+\hat{\bar{a}}_{2 I}^{2}+\hat{\bar{a}}_{3 I}^{2}+\hat{\bar{a}}_{4 I}^{2}, \\
d= & 2 \hat{\bar{a}}_{5 R} \hat{\bar{a}}_{7 R}+2 \hat{\bar{a}}_{6 R} \hat{\bar{a}}_{8 R}+2 \hat{\bar{a}}_{1 I} \hat{\bar{a}}_{3 I}+2 \hat{\bar{a}}_{2 I} \hat{\bar{a}}_{4 I} .
\end{array}
$$

From (12) we observe that inter-symbol-interference occurs

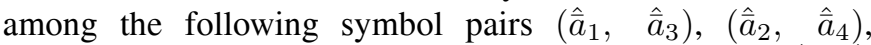
$\left(\hat{\bar{a}}_{5}, \hat{\bar{a}}_{7}\right)$, and $\left(\hat{\bar{a}}_{6}, \hat{\bar{a}}_{8}\right)$. In (9), since multiplying $\hat{\mathbf{X}}^{H} \hat{\mathbf{X}}$ by $\mathbf{h}_{j}^{H}$ and $\mathbf{h}_{j}$ does not create any additional crossterms between the symbols $\hat{\bar{a}}_{k}$, ML-decoding of the symbol vector a can be reduced to jointly decoding the symbol pairs $\left\{\left(\hat{\bar{a}}_{1}, \hat{\bar{a}}_{3}\right),\left(\hat{\bar{a}}_{2}, \hat{\bar{a}}_{4}\right),\left(\hat{\bar{a}}_{5}, \hat{\bar{a}}_{7}\right),\left(\hat{\bar{a}}_{6}, \hat{\bar{a}}_{8}\right)\right\}$ independently. Since $\hat{\bar{a}}_{k}$ is obtained by rotating the constellation of $\hat{a}_{k}, k=1, \ldots, 8$, decoding the symbols pairs $\left\{\left(\hat{a}_{1}, \hat{a}_{3}\right),\left(\hat{a}_{2}, \hat{a}_{4}\right),\left(\hat{a}_{5}, \hat{a}_{7}\right),\left(\hat{a}_{6}, \hat{a}_{8}\right)\right\}$ independently is MLoptimum. For the 4-transmit-antenna case we replace $\mathbf{H}$ by $\mathbf{H}_{e}$ and $\mathbf{Y}$ by $\mathbf{Y}_{e}$ in (9) to obtain a similar result. Since both $\mathbf{Y}_{1}$ and $\mathbf{Y}_{2}$ are required for optimal decoding, the proposed algorithm decodes every eight slots as opposed to four slots for conventional 4-transmit-antenna schemes.

As a concluding remark, we can show that our suggested code structure in (4) for the 8-transmit-antenna case is equivalent to the one in [13]. However, we do favor the code in (4) for two reasons: (i) Its simplicity in code construction enables our theoretical analysis on rotation angle optimization and, (ii) unlike the codewords in [13], [14], the codeword in (4) may be applied to a 4-transmit-antenna system across eight timeslots without omitting any symbols. Having shown already that the code supports ML-optimal two-symbol decoding, we now evaluate its diversity order and seek the rotation angles that improve error-rate performance.

\section{Diversity Order CAlculation: Eight Transmit ANTENNAS}

The probability of receiving the codeword $\widetilde{\mathbf{X}}$ when $\mathbf{X} \neq$ $\widetilde{\mathbf{X}}$ is transmitted is upper bounded by [17]

$$
\operatorname{Pr}(\mathbf{X} \rightarrow \widetilde{\mathbf{X}}) \leq \frac{1}{2}\left(\prod_{i=1}^{R}\left\{\frac{1}{1+\frac{\rho^{2} A \lambda_{i}}{4 N_{t}}}\right\}\right)^{N_{r}}
$$

where $R$ is the rank of $(\mathbf{X}-\widetilde{\mathbf{X}})$ and $\lambda_{i}, i=1, \ldots, R$, are the eigenvalues of $(\mathbf{X}-\widetilde{\mathbf{X}})^{H}(\mathbf{X}-\widetilde{\mathbf{X}})$. The minimum value of $R$ obtained over all codeword pairs is called the diversity order of the system [17] and dictates the slope of the errorrate curves at asymptotically high SNR values. To evaluate the diversity order of the codeword in (4), we seek the eigenvalues of $(\mathbf{X}-\widetilde{\mathbf{X}})^{H}(\mathbf{X}-\widetilde{\mathbf{X}})$. Set $\Delta \mathbf{X} \triangleq \mathbf{X}-\widetilde{\mathbf{X}}$ and $\Delta \mathbf{x}_{k} \triangleq \mathbf{x}_{k}-$ $\widetilde{\mathbf{x}}_{k}, k=1, \ldots, 8 ;$ then,

$$
\Delta \mathbf{X}^{H} \Delta \mathbf{X}=\left[\begin{array}{cccc}
\Delta a \mathbf{I}_{2} & \Delta b \mathbf{I}_{2} & \multicolumn{2}{c}{\mathbf{0}} \\
\Delta b \mathbf{I}_{2} & \Delta a \mathbf{I}_{2} & \Delta c \mathbf{I}_{2} & \Delta d \mathbf{I}_{2} \\
0 & \Delta d \mathbf{I}_{2} & \Delta c \mathbf{I}_{2}
\end{array}\right]
$$


where

$$
\begin{array}{ccc}
\Delta a \triangleq & \sum_{k=1}^{K}\left|\Delta x_{k}\right|^{2}, \\
\Delta b \triangleq & \sum_{k=1}^{K / 2} 2 \operatorname{Re}\left\{\Delta x_{k}^{*} \Delta x_{k+K / 2}\right\}, \\
\Delta c \triangleq & \sum_{k=K+1}^{2 K}\left|\Delta x_{k}\right|^{2}, \\
\Delta d \triangleq & \sum_{k=K+1}^{K+K / 2} 2 \operatorname{Re}\left\{\Delta x_{k}^{*} \Delta x_{k+K / 2}\right\}, \quad K=4 .
\end{array}
$$

The block diagonal nature of $\Delta \mathrm{X}^{H} \Delta \mathrm{X}$ in (14) allows us to calculate its eigenvalues as $\{(\Delta a-\Delta b),(\Delta a+\Delta b)$, $(\Delta c-\Delta d),(\Delta c+\Delta d)\}$ which exist with multiplicity of two. Expanding and simplifying, the eigenvalues are as shown below:

$$
\begin{aligned}
\left\{\left(\Delta \bar{a}_{1 R}-\Delta \bar{a}_{3 R}\right)^{2}+\right. & \left(\Delta \bar{a}_{2 R}-\Delta \bar{a}_{4 R}\right)^{2}+\left(\Delta \bar{a}_{5 I}-\Delta \bar{a}_{7 I}\right)^{2} \\
& +\left(\Delta \bar{a}_{6 I}-\Delta \bar{a}_{8 I}\right)^{2} \\
\left(\Delta \bar{a}_{1 R}+\Delta \bar{a}_{3 R}\right)^{2}+ & \left(\Delta \bar{a}_{2 R}+\Delta \bar{a}_{4 R}\right)^{2}+\left(\Delta \bar{a}_{5 I}+\Delta \bar{a}_{7 I}\right)^{2} \\
& +\left(\Delta \bar{a}_{6 I}+\Delta \bar{a}_{8 I}\right)^{2} \\
\left(\Delta \bar{a}_{5 R}-\Delta \bar{a}_{7 R}\right)^{2}+ & \left(\Delta \bar{a}_{6 R}-\Delta \bar{a}_{8 R}\right)^{2}+\left(\Delta \bar{a}_{1 I}-\Delta \bar{a}_{3 I}\right)^{2} \\
& +\left(\Delta \bar{a}_{2 I}-\Delta \bar{a}_{4 I}\right)^{2} \\
\left(\Delta \bar{a}_{5 R}+\Delta \bar{a}_{7 R}\right)^{2}+ & \left(\Delta \bar{a}_{6 R}+\Delta \bar{a}_{8 R}\right)^{2}+\left(\Delta \bar{a}_{1 I}+\Delta \bar{a}_{3 I}\right)^{2} \\
& \left.+\left(\Delta \bar{a}_{2 I}+\Delta \bar{a}_{4 I}\right)^{2}\right\} .
\end{aligned}
$$

As each eigenvalue is a summation of squares, without loss of generality suppose $\Delta \bar{a}_{1 R}, \Delta \bar{a}_{1 I}, \Delta \bar{a}_{3 R}, \Delta \bar{a}_{3 I} \neq 0$, the set of minimum eigenvalues over all possible codewords pairs is

$$
\begin{gathered}
\left\{\left(\Delta \bar{a}_{1 R}-\Delta \bar{a}_{3 R}\right)^{2},\left(\Delta \bar{a}_{1 R}+\Delta \bar{a}_{3 R}\right)^{2},\left(\Delta \bar{a}_{1 I}-\Delta \bar{a}_{3 I}\right)^{2},\right. \\
\left.\left(\Delta \bar{a}_{1 I}+\Delta \bar{a}_{3 I}\right)^{2}\right\}
\end{gathered}
$$

and represents the worst case scenario for the upper bound in (13). As long as all eigenvalues in (17) are non-zero, the codeword $\mathbf{X}$ achieves the maximum diversity order of 8 .

We now attempt to identify conditions on the rotation angles that have to be satisfied to allow the codeword $\mathbf{X}$ to achieve full diversity order. It is easily observed that all the eigenvalues in (17) are non-zero if and only if the product of the eigenvalues in (17) is non-zero, which in turn is the square root of the minimum value of the determinant of the $\Delta \mathbf{X}^{H} \Delta \mathbf{X}$. We have,

$$
\begin{aligned}
\min \operatorname{det} & \left(\Delta \mathbf{X}^{H} \Delta \mathbf{X}\right) \\
= & {\left[\left(\Delta \bar{a}_{1 R}-\Delta \bar{a}_{3 R}\right)^{4}\left(\Delta \bar{a}_{1 R}+\Delta \bar{a}_{3 R}\right)^{4}\right.} \\
& \left.\left(\Delta \bar{a}_{1 I}-\Delta \bar{a}_{3 I}\right)^{4}\left(\Delta \bar{a}_{1 I}+\Delta \bar{a}_{3 I}\right)^{4}\right] \\
= & {\left[\left(\Delta \bar{a}_{1 R}^{2}-\Delta \bar{a}_{3 R}^{2}\right)\left(\Delta \bar{a}_{1 I}^{2}-\Delta \bar{a}_{3 I}^{2}\right)\right]^{4} } \\
= & {\left[\begin{array}{c}
{\left[\left(\Delta a_{1 R} \cos (\phi)-\Delta a_{1 I} \sin (\phi)\right)^{2}\right.} \\
\left.-\left(\Delta a_{3 R} \cos (\theta)-\Delta a_{3 I} \sin (\theta)\right)^{2}\right] \\
\times\left[\left(\Delta a_{1 R} \sin (\phi)+\Delta a_{1 I} \cos (\phi)\right)^{2}\right. \\
\left.-\left(\Delta a_{3 R} \sin (\theta)+\Delta a_{3 I} \cos (\theta)\right)^{2}\right]
\end{array}\right] }
\end{aligned}
$$

For fixed $\phi, \theta$, we categorize (18) into five cases depending on the values of $\Delta a_{1 R}, \Delta a_{3 R}, \Delta a_{1 I}, \Delta a_{3 I}$. Note that we cannot have $\Delta a_{1 R}=\Delta a_{3 R}=\Delta a_{1 I}=\Delta a_{3 I}=0$.

1) Only one non-zero value (for example $\Delta a_{3 R}=$ $\left.\Delta a_{1 I}=\Delta a_{3 I}=0, \Delta a_{1 R} \neq 0\right)$ :

$$
\min \operatorname{det}\left(\Delta \mathbf{X}^{H} \Delta \mathbf{X}\right)=\left[\left(\Delta a_{1 R} \cos (\phi)\right)^{2}\left(\Delta a_{1 R} \sin (\phi)\right)^{2}\right]^{4} .
$$

If any of $\cos (\phi), \sin (\phi), \cos (\theta), \sin (\theta)$ is zero, full diversity order is not achieved.
2) Two non-zero values both from same symbol (for example $\Delta a_{3 R}=\Delta a_{3 I}=0, \Delta a_{1 R}, \Delta a_{1 I} \neq 0$ ):

$$
\begin{aligned}
\min \operatorname{det}\left(\Delta \mathbf{X}^{H} \Delta \mathbf{X}\right)= & {\left[\left(\left(\Delta a_{1 R} \cos (\phi)-\Delta a_{1 I} \sin (\phi)\right)^{2}\right)\right.} \\
& \left.\cdot\left(\left(\Delta a_{1 R} \sin (\phi)+\Delta a_{1 I} \cos (\phi)\right)^{2}\right)\right]^{4}
\end{aligned}
$$

Let $\mathcal{A}$ denote the constellation of the symbols $a_{k}, k=1, \ldots, K$, and $\mathcal{A}_{\phi}, \mathcal{A}_{\theta}$ denote the constellations of symbols formed by rotating $\mathcal{A}$ by $\phi$ and $\theta$, respectively. If any two symbols chosen from $\mathcal{A}_{\phi}$ or $\mathcal{A}_{\theta}$ have the same real part or the same imaginary part, full diversity order cannot be achieved.

3) Two non-zero values both either real or imaginary (for example $\Delta a_{1 I}=\Delta a_{3 I}=0, \Delta a_{1 R}, \Delta a_{3 R} \neq 0$ ):

$$
\begin{aligned}
\min \operatorname{det}\left(\Delta \mathbf{X}^{H} \Delta \mathbf{X}\right)= & \left(\left[\left(\Delta a_{1 R} \cos (\phi)\right)^{2}-\left(\Delta a_{3 R} \cos (\theta)\right)^{2}\right]\right. \\
& \left.\cdot\left[\left(\Delta a_{1 R} \sin (\phi)\right)^{2}-\left(\Delta a_{3 R} \sin (\theta)\right)^{2}\right]\right)^{4} .
\end{aligned}
$$

If $\theta=\phi$, full diversity order cannot be achieved.

4) Two non-zero values, one from real part of one symbol and other from imaginary part of another symbol ( for example $\Delta a_{1 I}=\Delta a_{3 R}=0, \Delta a_{1 R}, \Delta a_{3 I} \neq 0$ ):

$$
\begin{aligned}
\min \operatorname{det}\left(\Delta \mathbf{X}^{H} \Delta \mathbf{X}\right)= & \left(\left[\left(\Delta a_{1 R} \cos (\phi)\right)^{2}-\left(\Delta a_{3 I} \sin (\theta)\right)^{2}\right]\right. \\
& \left.\cdot\left[\left(\Delta a_{1 R} \sin (\phi)\right)^{2}-\left(\Delta a_{3 I} \cos (\theta)\right)^{2}\right]\right)^{4} .
\end{aligned}
$$

If $\theta=\phi \pm \pi / 2$, full diversity order cannot be achieved.

5) All values are non-zero (that is $\left.\Delta a_{1 I}, \Delta a_{3 I}, \Delta a_{1 R}, \Delta a_{3 R} \neq 0\right)$ :

If the square distance between the real parts of any two symbols from $\mathcal{A}_{\phi}$ is equal to the square distance between the real parts of any two symbols from $\mathcal{A}_{\theta}$ or the square distance between the imaginary parts of any two symbols from $\mathcal{A}_{\phi}$ is equal to the square distance between the imaginary parts of any two symbols from $\mathcal{A}_{\theta}$, full diversity order is not achieved.

For a given constellation, several pairs of $\phi, \theta$ may exist that satisfy the exclusion conditions listed above. In the next section, we investigate four different criteria for rotation angle optimization.

\section{Rotation Angle Optimization: Eight Transmit ANTENNAS}

We now consider four different criteria to optimize the rotation angles and for each criterion, we show how $\phi, \theta$ can be obtained for the codeword in (4) using (17). For ease in reference, our findings (values of $\phi, \theta$ for each criterion) are summarized in Table I.

\section{A. Diversity product maximization}

At high SNR values assuming full transmit diversity order and $N_{r}=1$, (13) can be approximated by

$$
\operatorname{Pr}(\mathbf{X} \rightarrow \widetilde{\mathbf{X}}) \leq \frac{1}{2}\left(\prod_{i=1}^{N_{t}} \lambda_{i}\right)^{-1}\left(-\frac{A}{4 N_{t}}\right)^{-N_{t}}
$$

Worst-case minimization of the bound in (23) is equivalent to maximization of the minimum product of the eigenvalues 
(determinant of $\Delta \mathbf{X}^{H} \Delta \mathbf{X}$ ) over all possible codeword pairs, which in turn is commonly represented by the diversity product $\zeta$,

$$
\zeta=\frac{1}{2 \sqrt{N_{t}}} \min _{\mathbf{X} \neq \tilde{\mathbf{X}}}\left|\operatorname{det}\left[\Delta \mathbf{X} \Delta \mathbf{X}^{H}\right]\right|^{1 /(2 T)} .
$$

Diversity product maximization was used as the rotation angle design criterion in [13], [14]. For the codeword in (4), the minimum determinant of $\Delta \mathbf{X}^{H} \Delta \mathbf{X}$ over all codeword pairs is

$$
\begin{aligned}
\min \operatorname{det}\left(\Delta \mathbf{X}^{H} \Delta \mathbf{X}\right)= & {\left[\left(\Delta a_{1 R} \cos (\phi)-\Delta a_{1 I} \sin (\phi)\right)^{2}\right.} \\
& \left.-\left(\Delta a_{3 R} \cos (\theta)-\Delta a_{3 I} \sin (\theta)\right)^{2}\right]^{4} \\
\times & {\left[\left(\Delta a_{1 R} \sin (\phi)+\Delta a_{1 I} \cos (\phi)\right)^{2}\right.} \\
& \left.-\left(\Delta a_{3 R} \sin (\theta)+\Delta a_{3 I} \cos (\theta)\right)^{2}\right]^{4}(25)
\end{aligned}
$$

in which parameters $\phi$ and $\theta$ should be chosen to maximize (25). We evaluate and conclude (see Table I) that the codeword in (4) (and the codewords in [13], [14]) achieve diversity product of 0.1747 with 4-QAM constellation and 0.1071 with the non-rectangular 8-QAM constellation for the 8-transmitantenna case.

\section{B. Minimum sum-of-eigenvalues maximization}

In [18], it was proposed that for high diversity order systems (number of transmit antennas greater than four) the minimum trace of $\Delta \mathbf{X}^{H} \Delta \mathbf{X}$ is to be maximized over all codeword pairs. From our expression (16), we observe that

$$
\begin{aligned}
\min ( & \left.\operatorname{Tr}\left(\Delta \mathbf{X}^{H} \Delta \mathbf{X}\right)\right) \\
= & 2\left[\left(\Delta \bar{a}_{1 R}-\Delta \bar{a}_{3 R}\right)^{2}+\left(\Delta \bar{a}_{1 R}+\Delta \bar{a}_{3 R}\right)^{2}\right. \\
& \left.\quad+\left(\Delta \bar{a}_{1 I}-\Delta \bar{a}_{3 I}\right)^{2}+\left(\Delta \bar{a}_{1 I}+\Delta \bar{a}_{3 I}\right)^{2}\right] \\
= & 4\left[\Delta \bar{a}_{1 R}^{2}+\Delta \bar{a}_{3 R}^{2}+\Delta \bar{a}_{1 I}^{2}+\Delta \bar{a}_{3 I}^{2}\right] \\
= & 4\left[\Delta a_{1 R}^{2}+\Delta a_{3 R}^{2}+\Delta a_{1 I}^{2}+\Delta a_{3 I}^{2}\right]
\end{aligned}
$$

which is independent of $\phi, \theta$. Hence, for the proposed codeword and that of [13], [14], the sum-of-eigenvalues criterion in [18] is not relevant.

\section{Minimum eigenvalue maximization}

If $r<N_{t}$ eigenvalues of $\Delta \mathbf{X}^{H} \Delta \mathbf{X}$ are significantly less than 1 , then even for large SNR values, $1+\frac{A \lambda_{i}}{4 N_{t}} \simeq 1$ and $(13)$ is approximated by

$$
\operatorname{Pr}(\mathbf{X} \rightarrow \widetilde{\mathbf{X}}) \leq \frac{1}{2}\left(\prod_{i=1}^{N_{t}-r} \lambda_{i}\right)^{-1}\left(-\frac{A}{4 N_{t}}\right)^{-\left(N_{t}-r\right)}
$$

The system seems to lose diversity; for the codeword in (4) and the proposed codewords in [13], [14] the occurrence of the eigenvalues in pairs causes loss of diversity in steps of two. In such circumstances, it appears reasonable to consider rotation angle choices that maximize the minimum possible eigenvalue over all pairs of codewords. For our code structure in (4), the rotation angles that maximize the minimum eigenvalue are

$$
\begin{aligned}
& (\phi, \theta)=\underset{\phi, \theta}{\operatorname{argmax}} \min \left\{\left(\Delta \bar{a}_{1 R}-\Delta \bar{a}_{3 R}\right)^{2},\right. \\
& \left.\quad\left(\Delta \bar{a}_{1 R}+\Delta \bar{a}_{3 R}\right)^{2},\left(\Delta \bar{a}_{1 I}-\Delta \bar{a}_{3 I}\right)^{2},\left(\Delta \bar{a}_{1 I}+\Delta \bar{a}_{3 I}\right)^{2}\right\} .
\end{aligned}
$$

The solution is listed in Table I.

\section{PEP-bound Minimization}

We now show that for all STBCs that employ CR and have no more than two unique eigenvalues of equal multiplicity of $\Delta \mathbf{X}^{H} \Delta \mathbf{X}$ over all possible codeword pairs, maximization of the diversity product is equivalent to minimization of the upper bound on PEP for all SNRs. Minimization of the bound in (13) is equivalent to maximizing $\prod_{i=1}^{R}\left\{1+\frac{A \lambda_{i}}{4 N_{t}}\right\}$. If $\lambda_{1}$ and $\lambda_{2}$ represent the 2 unique eigenvalues of $\Delta \mathbf{X}^{H} \Delta \mathbf{X}$, then

$$
\begin{aligned}
& \operatorname{argmax} \prod_{i=1}^{R}\left(1+\frac{A \lambda_{i}}{4 N_{t}}\right) \\
& \quad=\operatorname{argmax}\left[\left(1+\frac{A \lambda_{1}}{4 N_{t}}\right)\left(1+\frac{A \lambda_{2}}{4 N_{t}}\right)\right]^{q} \\
& \quad=\operatorname{argmax}\left[1+\frac{A}{4 N_{t}}\left(\lambda_{1}+\lambda_{2}\right)+\frac{A^{2}}{16 N_{t}^{2}}\left(\lambda_{1} \lambda_{2}\right)\right]^{q}
\end{aligned}
$$

where $q$ denotes the multiplicity of the eigenvalues. Since $q\left(\lambda_{1}+\lambda_{2}\right)=\operatorname{tr}\left(\Delta \mathbf{X}^{H} \Delta \mathbf{X}\right)=\|\Delta \mathbf{X}\|_{F}^{2}$ is independent of rotation angles ${ }^{1}$ we need to maximize only the product of the eigenvalues to minimize the bound in (13). In the case of a single unique eigenvalue (as in O-STBCs for example), the STBC is independent of the rotation angle.

While the case of two unique eigenvalues of equal multiplicity applies to the $4 \times 4$ QO-STBCs proposed in [7], [10], [11] and their choice of rotation angle is PEP-bound optimal, for the $8 \times 8$ codewords four unique eigenvalues exist and maximizing the eigenvalue (diversity) product over all possible codeword pairs does not necessarily minimize the maximum bound in (13).

We now directly find the rotation angles that minimize the maximum (worst case) PEP-upper-bound. Substitution of (17) in (13) gives us the worst case scenario for all codeword pairs. We need to optimize $\phi, \theta$ such that

$$
(\phi, \theta)=\underset{\phi, \theta}{\operatorname{argmax}} \prod_{i=1}^{4}\left[\left(1+\frac{A \lambda_{i}}{4 N_{t}}\right)\right]^{2}
$$

where $\quad \lambda_{i} \in\left\{\left(\Delta \bar{a}_{1 R}-\Delta \bar{a}_{3 R}\right)^{2},\left(\Delta \bar{a}_{1 R}+\Delta \bar{a}_{3 R}\right)^{2}\right.$, $\left.\left(\Delta \bar{a}_{1 I}-\Delta \bar{a}_{3 I}\right)^{2},\left(\Delta \bar{a}_{1 I}+\Delta \bar{a}_{3 I}\right)^{2}\right\}, \quad i=1,2,3,4$. Suitable values for $A$ can be chosen such that $A \lambda_{i}>1$ for all $i=1,2,3,4$. The optimized angles are listed in Table I.

\section{Diversity ORder CALCUlation AND Rotation ANgLE Optimization: Four TRANSmit Antennas}

In this section, we aim to extend the results that we have obtained for the 8-transmit-antenna case to the correlated 4 -transmit-antenna system. For clarity in presentation we would like to reiterate our transmission scheme for 4-transmitantennas. We transmit $\mathbf{X}_{1}$ in the first four time-slots and then $\mathbf{X}_{2}$ in the next four time slots. Upon reception of both $\mathbf{Y}_{1}$ and $\mathbf{Y}_{2}$, the ML estimate of the transmitted signal vector $\mathbf{a}$ is obtained by joint two symbol decoding as described in Section II. We now aim to show that this transmission scheme has a diversity order of 8 .

To proceed we need to reevaluate the PEP-upper-bound in (13) due to the correlation that exists between elements of $\mathbf{H}_{1}$ and $\mathbf{H}_{2}$. The new PEP-upper-bound can be obtained either by considering the time-correlated channel model in (6) or 
TABLE I

OPTIMAL ANGLES

\begin{tabular}{|c|c|c|c|c|}
\hline QAM & Criterion & $(\phi, \theta)$ & Diversity Product & Min. Eigenvalue \\
\hline \hline 4 & Diversity Product & $(37.9,21.4)$ & 0.1747 & 0.0093 \\
\hline 4 & Min. Eigenvalue & $(30.9,13.3)$ & 0.1623 & 0.0524 \\
\hline 4 & Max. PEP Bound & $(28.5,40)$ & 0.1352 & 0.0112 \\
\hline 8 & Diversity Product & $\left(\tan ^{-1}(2) / 2, \tan ^{-1}(1 / 2) / 2\right)$ & 0.1071 & $2.35 \times 10^{-4}$ \\
\hline 8 & Min. Eigenvalue & $\left(3, \tan ^{-1}(2)\right)$ & 0.0732 & 0.0022 \\
\hline 8 & Max. PEP Bound & $(7.2,25.1)$ & 0.0792 & $2.7 \times 10^{-4}$ \\
\hline
\end{tabular}

the space-correlated channel model in (8). We begin with the time-correlated model in (6).

Defining $\mathbf{X}_{t} \triangleq\left[\mathbf{X}_{1}^{T} \mathbf{X}_{2}^{T}\right]^{T}$ and $\Delta \mathbf{X}_{t} \triangleq \mathbf{X}_{t}-\widetilde{\mathbf{X}}_{t}$ where $\mathbf{X}_{t} \neq \widetilde{\mathbf{X}}_{t}$, the PEP for a time-correlated fast fading channel (channel coefficients changing every time slot) is upperbounded by [19]

$$
\operatorname{Pr}\left(\mathbf{X}_{t} \rightarrow \widetilde{\mathbf{X}}_{t}\right) \leq\left(\begin{array}{c}
2 r_{t} N_{r}-1 \\
r_{t} N_{r}
\end{array}\right)\left(\prod_{i=1}^{r_{t}} \Lambda_{t i}\right)^{-N_{r}}\left(\frac{A}{N_{t}}\right)^{-r_{t} N_{r}}
$$

where $r_{t}$ is the rank of the matrix $\left(\Delta \mathbf{X}_{t} \Delta \mathbf{X}_{t}^{H}\right) \circ \mathbf{R}_{t}$ ( is the Hadamard product operator), $\Lambda_{t i}, i=1, \ldots, r_{t}$, are the non-zero eigenvalues of $\left(\Delta \mathbf{X}_{t} \Delta \mathbf{X}_{t}^{H}\right) \circ \mathbf{R}_{t}$, and $\mathbf{R}_{t}$ is the time-correlated channel matrix to be evaluated. Conforming with the signal model in [19], with $T=4$ and $N_{r}=1$ the received signal vector $\mathbf{y}_{e 1} \triangleq\left[\mathbf{y}_{1}^{T} \mathbf{y}_{2}^{T}\right]^{T}\left(\mathbf{y}_{1}\right.$ and $\mathbf{y}_{2}$ are the first columns of $\mathbf{Y}_{1}, \mathbf{Y}_{2}$ in (6)) is

$$
\mathbf{y}_{e 1}=\sqrt{\frac{A}{N_{t}}}\left[\mathbf{D}_{1}, \ldots, \mathbf{D}_{N_{t}}\right] \mathbf{h}_{t}+\mathbf{n}_{t}
$$

where $\mathbf{D}_{i}=\operatorname{diag}\left\{\mathbf{x}_{t i}\right\}$ are the $2 T \times 2 T$ matrices created by the columns of $\mathbf{X}_{t}, \mathbf{x}_{t i}, i=1,2, \cdots, N_{t}$. The $2 T \times 1$ noise vector $\mathbf{n}_{t}$ satisfies $E\left\{\mathbf{n}_{t} \mathbf{n}_{t}^{H}\right\}=\mathbf{I}_{2 T}$. The $2 T N_{t} \times 1$ channel vector is $\mathbf{h}_{t}=\left[\mathbf{h}_{t 1}^{T}, \ldots, \mathbf{h}_{t N_{t}}^{T}\right]^{T}$ where $\mathbf{h}_{t i}$ is the vector corresponding to the $i t h, i=1, \ldots, N_{t}$, transmit antenna. If $h_{e_{i, j}}, i=1, \ldots, 2 N_{t}, j=1, \ldots, N_{r}$, represent the elements of $\mathbf{H}_{e}=\left[\begin{array}{ll}\mathbf{H}_{1}^{T} & \mathbf{H}_{2}^{T}\end{array}\right]^{T}$, then $\mathbf{h}_{t i}=$ $\left[h_{e_{i, 1}} h_{e_{i, 1}} h_{e_{i, 1}} h_{e_{i, 1}} \quad h_{e_{i+N_{t}, 1}} h_{e_{i+N_{t}, 1}} h_{e_{i+N_{t}, 1}} h_{e_{i+N_{t}, 1}}\right]^{T}$. The time-correlated channel matrix $\mathbf{R}_{t}$ can now be defined as

$$
\mathbf{R}_{t} \triangleq E\left\{\mathbf{h}_{t i} \mathbf{h}_{t i}^{H}\right\}=\left[\begin{array}{cc}
\mathbf{1}_{4} & p \mathbf{1}_{4} \\
p \mathbf{1}_{4} & \mathbf{1}_{4}
\end{array}\right], i=1, \ldots, N_{t}
$$

where $\mathbf{1}_{4}$ is a $4 \times 4$ all-one matrix. Having evaluated $\mathbf{R}_{t}$, we calculate

$$
\begin{aligned}
& \left(\Delta \mathbf{X}_{t} \Delta \mathbf{X}_{t}^{H}\right) \circ \mathbf{R}_{t} \\
& =\left[\begin{array}{cc}
\Delta \mathbf{X}_{1} \Delta \mathbf{X}_{1}^{H} & p \Delta \mathbf{X}_{1} \Delta \mathbf{X}_{2}^{H} \\
p \Delta \mathbf{X}_{2} \Delta \mathbf{X}_{1}^{H} & \Delta \mathbf{X}_{2} \Delta \mathbf{X}_{2}^{H}
\end{array}\right] \\
& =\left[\begin{array}{cc}
\Delta \mathbf{X}_{1} & \mathbf{0}_{4} \\
\mathbf{0}_{4} & \Delta \mathbf{X}_{2}
\end{array}\right] \cdot\left[\begin{array}{cc}
\mathbf{I}_{4} & p \mathbf{I}_{4} \\
p \mathbf{I}_{4} & \mathbf{I}_{4}
\end{array}\right] \cdot\left[\begin{array}{cc}
\Delta \mathbf{X}_{1}^{H} & \mathbf{0}_{4} \\
\mathbf{0}_{4} & \Delta \mathbf{X}_{2}^{H}
\end{array}\right] \\
& =\Delta \mathbf{X R}_{s} \Delta \mathbf{X}^{H}
\end{aligned}
$$

with $\mathbf{R}_{s} \triangleq E\left\{\mathbf{h}_{e j} \mathbf{h}_{e l}^{H}\right\}=\left[\begin{array}{cc}\mathbf{1}_{4} & p \mathbf{1}_{4} \\ p \mathbf{1}_{4} & \mathbf{1}_{4}\end{array}\right]$ for $j=l$ from (7). Since $\operatorname{eig}\left(\mathbf{R}_{s} \Delta \mathbf{X}^{H} \Delta \mathbf{X}\right)=e i g\left(\Delta \mathbf{X R}_{s} \Delta \mathbf{X}^{H}\right)$ [20], expanding $\mathbf{R}_{s} \Delta \mathbf{X}^{H} \Delta \mathbf{X}$ we have
$\mathbf{R}_{s} \Delta \mathbf{X}^{H} \Delta \mathbf{X}=\left[\begin{array}{cccc}\Delta a \mathbf{I}_{2} & \Delta b \mathbf{I}_{2} & p \Delta c \mathbf{I}_{2} & p \Delta d \mathbf{I}_{2} \\ \Delta b \mathbf{I}_{2} & \Delta a \mathbf{I}_{2} & p \Delta d \mathbf{I}_{2} & p \Delta c \mathbf{I}_{2} \\ p \Delta a \mathbf{I}_{2} & p \Delta b \mathbf{I}_{2} & \Delta c \mathbf{I}_{2} & \Delta d \mathbf{I}_{2} \\ p \Delta b \mathbf{I}_{2} & p \Delta a \mathbf{I}_{2} & \Delta d \mathbf{I}_{2} & \Delta c \mathbf{I}_{2}\end{array}\right]$

This concludes the analysis work for the PEP-upper-bound for the time-correlated model in (31).

When we consider the space-correlated model in (8) we obtain the PEP-upper-bound as

$$
\operatorname{Pr}(\mathbf{X} \rightarrow \widetilde{\mathbf{X}}) \leq \frac{1}{2}\left(\prod_{i=1}^{r_{t}}\left\{\frac{1}{1+\frac{A \Lambda_{t i}}{4 N_{t}}}\right\}\right)^{N_{r}}
$$

where $r_{t}$ and $\Lambda_{t i}$ have the same definition as before.

The rank $r_{t}$ is the diversity order of the transmission scheme for the 4-transmit-antenna case and from (35) we observe that a maximum value of 8 is attainable provided the matrix $\mathbf{R}_{s} \Delta \mathbf{X}^{H} \Delta \mathbf{X}$ is full-rank. Due to the loss of the block diagonal structure (cf. (35)), individual eigenvalues of $\mathbf{R}_{s} \Delta \mathbf{X}^{H} \Delta \mathbf{X}$ do not yield simplified expressions as was the case for the 8transmit-antenna system. However, the product and sum of the eigenvalues can still be evaluated.

\section{A. Diversity product maximization}

We have

$$
\prod_{i=1}^{N_{t}} \Lambda_{t i}=\operatorname{det}\left(\mathbf{R}_{s} \Delta \mathbf{X}^{H} \Delta \mathbf{X}\right)=\operatorname{det}\left(\mathbf{R}_{s}\right) \operatorname{det}\left(\Delta \mathbf{X}^{H} \Delta \mathbf{X}\right)
$$

and the diversity product for the 4-transmit-antenna case is given by

$$
\begin{aligned}
\zeta & =\frac{1}{2 \sqrt{N_{t}}} \min _{\mathbf{X} \neq \tilde{\mathbf{X}}}\left|\operatorname{det}\left[\Delta \mathbf{X} \Delta \mathbf{X}^{H}\right] \operatorname{det}\left[\mathbf{R}_{s}\right]\right|^{1 /(2 T)} \\
& =\frac{\left(1-p^{2}\right)^{2 / T}}{2 \sqrt{N_{t}}} \min _{\mathbf{X} \neq \tilde{\mathbf{X}}}\left|\operatorname{det}\left[\Delta \mathbf{X} \Delta \mathbf{X}^{H}\right]\right|^{1 /(2 T)}
\end{aligned}
$$

We conclude that the rotation angles $\phi, \theta$ that maximize the diversity product for the uncorrelated 8-transmit-antenna case also maximize the diversity product for the correlated 4-transmit-antenna case (is independent of the correlation coefficient value). Since there exist angles $\phi, \theta$ for which (38) is non-zero (several examples are listed in Table I), $\mathbf{R}_{s} \Delta \mathbf{X}^{H} \Delta \mathbf{X}$ is full rank and the transmission scheme for 4 transmit-antennas achieves the maximum diversity $\operatorname{order}^{2}$ of 8. This might seem, arguably, surprising in the context of the

${ }^{2}$ However, due to the correlation between the corresponding elements of $\mathbf{H}_{1}$ and $\mathbf{H}_{2}$, the codeword $\mathbf{X}$ experiences a loss in diversity product by a factor of $\left(1-p^{2}\right)^{2 / T}(\mathrm{cf} .(38))$. 
findings in [19] where for fast fading time-correlated channels optimal diversity-product rotation angles are a function of the correlation coefficient $p$. We can also observe that when $p=1$, the diversity product in (38) becomes zero and the maximum achievable rank of $\mathbf{R}_{s} \Delta \mathbf{X}^{H} \Delta \mathbf{X}$ is $4(\Delta a=\Delta c$ and $\Delta b=\Delta d$ ) which is intuitively satisfying and expected as $\mathbf{X}_{2}$ experiences the same fading as $\mathbf{X}_{1}$.

\section{B. Minimum sum-of-eigenvalues maximization}

Substituting from (12), we have

$$
\begin{aligned}
\sum_{i=1}^{N_{t}} \Lambda_{t i} & =\operatorname{Tr}\left(\mathbf{R}_{s} \Delta \mathbf{X}^{H} \Delta \mathbf{X}\right)=2(\Delta a+\Delta c) \\
& =2 \sum_{i=i}^{8} \Delta \bar{a}_{i R}^{2}+\Delta \bar{a}_{i I}^{2}=2 \sum_{i=i}^{8} \Delta a_{i R}^{2}+\Delta a_{i I}^{2}
\end{aligned}
$$

which is independent of the rotation angles $\phi, \theta$. Hence, once again, maximization of the minimum sum of eigenvalues (criterion suggested in [18]) is irrelevant to this system. Obtaining $\phi, \theta$ under the other two criteria proposed in Section IV (minimum eigenvalue maximization and direct PEPbound minimization) requires knowledge of each individual eigenvalue of $\mathbf{R}_{s} \Delta \mathbf{X}^{H} \Delta \mathbf{X}$. Even though the eigenvalues can be evaluated, they yield complicated expressions that are functions of $p$.

As a concluding remark, a most important aspect of the proposed transmission scheme for the 4-transmit-antenna case is that it enables transmit diversity order of 8 with no additional physical hardware at the transmitter (4 antennas only) and requires only joint two-symbol decoding at the receiver. In the section below, we evaluate the error-rate performance of the codeword $\mathbf{X}$ along with the various criteria for rotation angle optimization proposed in Section IV.

\section{Simulation Studies}

We present and discuss simulation studies for the 4 and 8-transmit-antenna cases with $N_{r}=1$.

\section{A. Four Transmit Antennas}

To gauge the performance of the proposed $8 \times 8$ QO-STBC transmitted by 4 antennas only, we compare its error rate against the $4 \times 4$ QO-STBC codeword in [7]. To the best of our knowledge, for a system with 4 transmit antennas the code in [7] has the best error-rate performance of all QOSTBCs that jointly decode two symbols or less. Since our proposed codeword has diversity order of 8 and rate 1 over 8 time slots, we may compare against two different $4 \times 4$ QOSTBC transmission schemes. In one scenario, we transmit the $4 \times 4$ QO-STBC codeword over the first four time slots and then repeat the same codeword over the next four time slots to maintain diversity order of 8 with, however, code rate $1 / 2(4$ symbols over 8 time slots). In the second scenario, we transmit a $4 \times 4$ QO-STBC codeword over the first four time slots and then transmit a new $4 \times 4$ QO-STBC codeword over the next four time slots to maintain code rate of 1 ( 8 symbols over 8 time slots) with, however, diversity order 4 .

The elements of the channel matrices, $h_{1_{i, j}}$ in $\mathbf{H}_{1}$ and $h_{2_{i, j}}$ in $\mathbf{H}_{2}$, are correlated and generated as follows:

$$
\begin{gathered}
h_{1_{i, j}} \sim \mathcal{C N}(0,1) \quad \text { and } h_{2_{i, j}}=p h_{1_{i, j}}+\sqrt{1-p^{2}} z_{i, j}, \\
i=1, \ldots, N_{t}, j=1,
\end{gathered}
$$

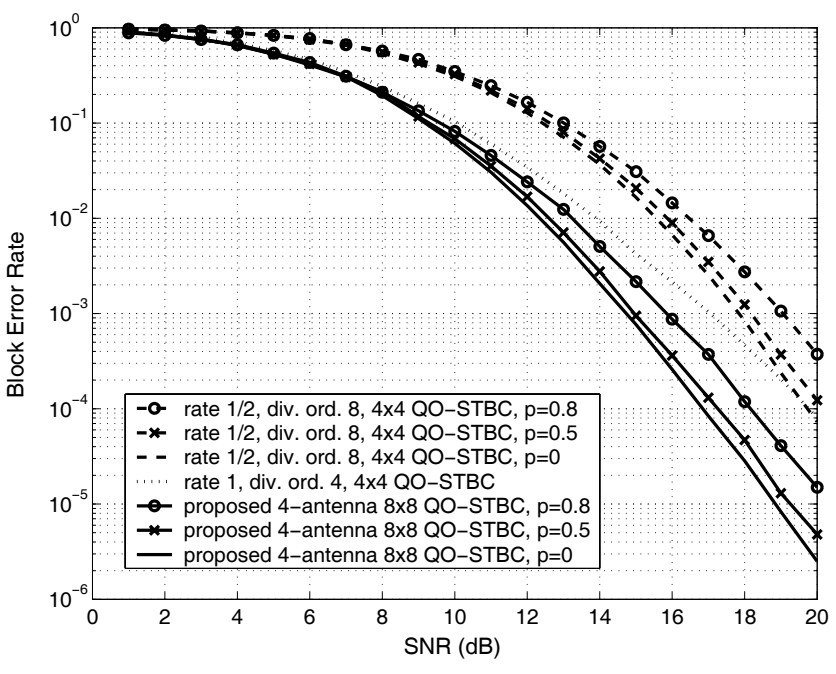

Fig. 1. Block-error-rate versus SNR (4-transmit-antenna system).

where $z_{i, j} \sim \mathcal{C N}(0,1)$ and $p$ is the correlation coefficient $(\mathcal{C N}$ stands for complex Gaussian distribution). In Fig. 1, we plot the block-error-rate as a function of the received signal-tonoise-ratio (SNR) for (i) our 4-antenna codeword transmission scheme in (3), (ii) transmission rate $1 / 2$, diversity order 8 transmission of the $4 \times 4$ QO-STBC of [7], and (iii) transmission rate 1 , diversity order $4,4 \times 4$ QO-STBC. We consider three channel fading correlation scenarios, $p=0, p=0.5$, and $p=0.8$. For rate 1 codewords, we select the symbols from a 4 QAM constellation, while for rate $1 / 2$ codewords the symbols are chosen from a 16-QAM constellation to maintain equal spectral efficiency for all transmission schemes. The rotation angles for our proposed codeword are $\{\phi, \theta\}=\{37.9,21.4\}$ under diversity product minimization, while for the $4 \times 4$ QO-STBC codeword the rotation angle is $\pi / 4$ as calculated in [7]. Since the $4 \times 4$ QO-STBC has only two unique eigenvalues, the angle of $\pi / 4$ is also PEP-bound optimal (not the case, however, for the proposed 4-transmit-antenna transmission scheme). From Fig. 1, we observe a substantial gain in performance achieved by transmitting the proposed $8 \times 8$ codeword over four antennas when compared with the two other $4 \times 4$ QO-STBC schemes. The best achievable performance from the $4 \times 4$ QO-STBC codeword of [7] is the direct rate 1 , diversity order 4 transmission, which the proposed transmission scheme outperforms handily even in the extreme scenario of $80 \%$ channel correlation. In fact, to that respect it may be worth noting that our matrices $\mathbf{X}_{1}$ and $\mathbf{X}_{2}$ may not need to be transmitted directly one after the other in time. Separating the transmissions of the two codeword blocks over several time-slot blocks (block interleaving) may help reduce the effective coefficient value of $p$ when allowed.

\section{B. Eight Transmit Antennas}

We now evaluate the performance of the $8 \times 8$ QO-STBC in (4) under 8-element antenna transmission and minimum eigenvalue $\mathrm{CR}$ optimization by (28), diversity product $\mathrm{CR}$ optimization by (25), and the proposed direct maximum PEPbound CR optimization by (30). In Fig. 2, we plot the blockerror-rate versus SNR when the symbols are chosen from a 


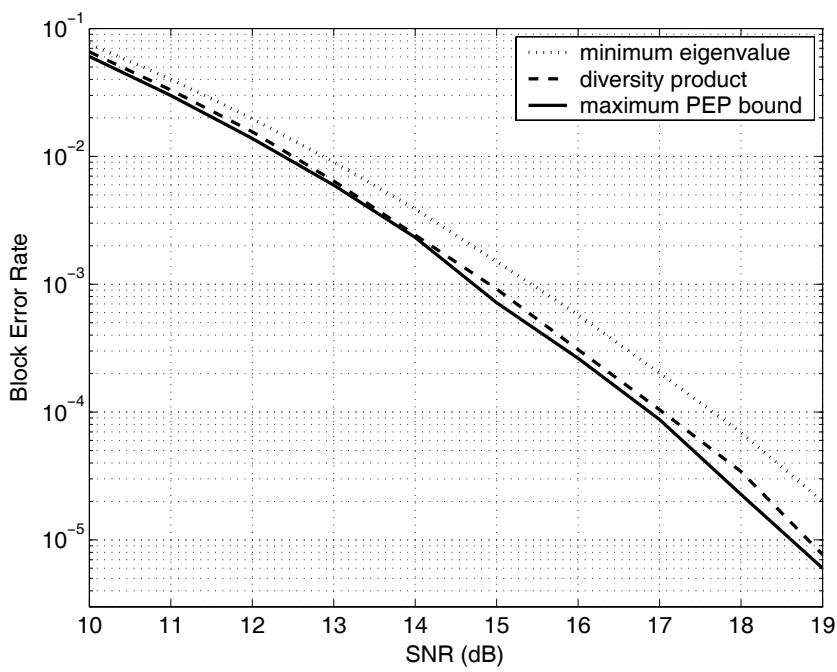

Fig. 2. Block-error-rate versus SNR for 4-QAM constellation (8-transmitantenna system).

4-QAM constellation. For direct PEP-bound optimization of $\phi, \theta$, we solve for $A$ to obtain a received SNR of $20 \mathrm{~dB}$. We observe that the rotation angles that maximize the diversity product and the rotation angles that minimize the maximum PEP bound provide the best results, with the latter having indeed better performance. The exact angle values are shown in Table I (along with the resulting diversity product and minimum eigenvalue).

In Fig. 3, we repeat the studies of Fig. 2 for symbols chosen from an 8-QAM non-rectangular constellation [8]. To obtain the PEP-bound optimal $\phi, \theta$, values we solve for $A$ that corresponds to received SNR of 30dB. Again, all calculated values are given in Table I. For reference purposes, we include in our comparisons the $8 \times 8$ single-symbol decodable STBC in [10]. Since the code in [10] contains only two unique eigenvalues the rotation angle of $\tan ^{-1}(1 / 2)$ is PEP-bound optimal for that code; since its rate is $3 / 4$, we select symbols from a 16-QAM constellation to ensure equal spectral efficiency for all codewords under comparison. Our PEP-bound optimized codeword in (4) offers a gain of about $1 \mathrm{~dB}$ over the single-symbol decodable STBC in [10]. The minimum eigenvalue optimized version performs almost similarly well. As argued in Section IV.C, due to decreased values of the minimum eigenvalues as compared to the 4-QAM scenario, the maximum diversity (eigenvalue) product optimized system seems to lose diversity over the operable SNR range. Similar performance loss was also observed in [13] when the rotation angles were chosen to maximize the diversity product.

\section{CONCLUSIONS}

We proposed an alternative representation of the $8 \times 8$ twosymbol decodable quasi-orthogonal space-time block code (QO-STBC) that can be used on both 8 and 4-transmit-antenna systems. For the 8-transmit-antenna system, we derived three different sets of rotation angle values: maximum diversityproduct optimal, maximum minimum-eigenvalue optimal, and minimum maximum-pairwise-error-probability-bound (PEP) optimal.

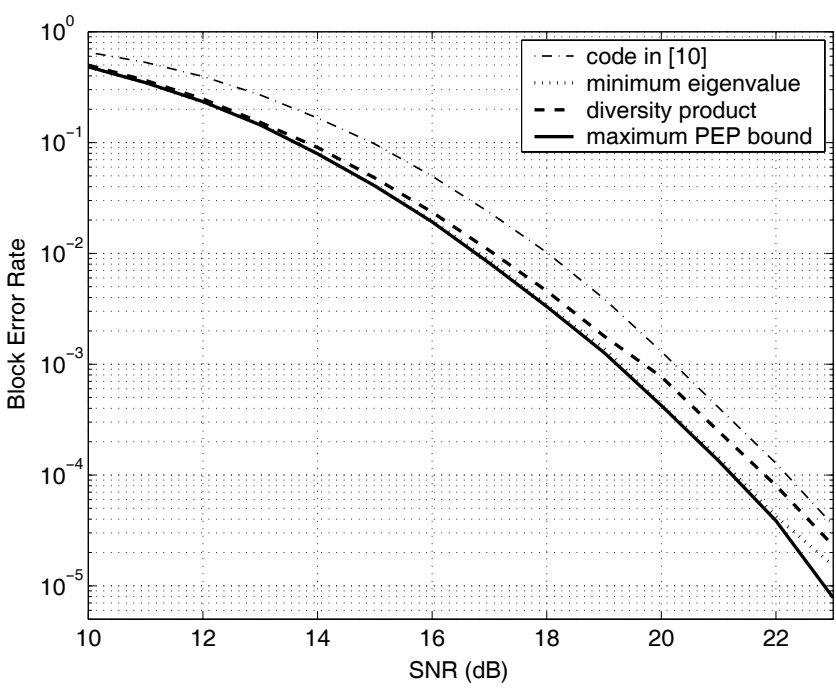

Fig. 3. Block-error-rate versus SNR for non-rectangular 8-QAM constellation and 16-QAM for the rate 3/4 code in [10] (8-transmit-antenna system).

Most importantly, the proposed codeword doubles the transmit diversity order of a 4-transmit-antenna system. By obtaining new expressions for the PEP-upper-bound for correlated channels we were able to find rotation angles that maximize the diversity product of 4 -antenna transmission systems and proved that the rotation angles are independent of the correlation coefficient. As a by-product, we showed that for the proposed codeword, maximization of the sum of eigenvalues is an irrelevant/non-applicable criterion (for both 8 and 4 -antenna transmissions).

\section{REFERENCES}

[1] S. Alamouti, "A simple transmit diversity technique for wireless communications," IEEE J. Select. Areas Commun., vol. 16, pp. 1451-1458, Oct. 1998.

[2] V. Tarokh, H. Jafarkhani, and A. R. Calderbank, "Space-time block codes from orthogonal designs," IEEE Trans. Inform. Theory, vol. 45, pp. 1456-1467, May 1999.

[3] W. Su, X. G. Xia, and K. J. R. Liu, "A systematic design of high-rate complex orthogonal space-time block codes," IEEE Commun. Lett., vol. 8, pp.380-382, June 2004.

[4] O. Tirkkonen, A. Boariu, and A. Hottinen, "Minimal non-orthogonality rate 1 space-time block code for 3+ Tx antennas," in Proc. IEEE 6th Int. Symp. Spread-Spectrum Techniques and Applications (ISSSTA 2000), Parsippany, NJ, Sept. 2000, pp. 429-432.

[5] H. Jafarkhani, "A quasi-orthogonal space-time block code," IEEE Trans. Commun., vol. 49, pp. 1-4, Jan. 2001.

[6] C. B. Papadias and G. J. Foschini, "Capacity-approaching space-time codes for systems employing four transmitter antennas," IEEE Trans. Inform. Theory, vol. 49, pp. 726-732, Mar. 2003.

[7] W. Su and X. G. Xia, "Quasi-orthogonal space-time block codes with full diversity," in Proc. IEEE Global Telecommun. Conf. (GLOBECOM), Taipei, Taiwan, Nov. 2002, vol. 2, pp. 1098-1102.

[8] W. Su and X. G. Xia, "Signal constellations for quasi-orthogonal spacetime block codes with full diversity," IEEE Trans. Inform. Theory, vol. 50, pp. 2331-2347, Oct. 2004.

[9] N. Sharma and C. B. Papadias, "Improved quasi-orthogonal codes through constellation rotation," IEEE Trans. Commun., vol. 51, pp. 332335, Mar. 2003.

[10] Z. A. Khan, B. S. Rajan, and M. H. Lee, "Rectangular co-ordinate interleaved orthogonal designs," in Proc. IEEE GLOBECOM, San Francisco, CA, Dec. 2003, vol. 4, pp. 2004-2009.

[11] C. Yuen, Y. L. Guan, and T. T. Tjhung, "Quasi-orthogonal STBC with minimum decoding complexity," IEEE Trans. Wireless Commun., vol. 4, pp. 2089-2094, Sept. 2005. 
[12] N. Sharma and C. B. Papadias, "Full rate full diversity linear quasiorthogonal space-time codes for any transmit antennas," EURASIP J. Applied Signal Proc., pp. 1246-1256, Aug. 2004.

[13] Z. A. Khan, M. H. Lee, and B. S. Rajan, "A rate-one fulldiversity quasi-orthogonal design for eight Tx antennas," Internal Report, TR-PME-2002-15, [Online]. Available: http://pal.ece.iisc.ernet.in/PAM/tech_rep02.html.

[14] C. Yuen, Y. L. Guan, and T. T. Tjhung, "A class of four-group quasiorthogonal STBC achieving full rate and full diversity for any number of antennas," in Proc. IEEE Intl. Symp. Personal, Indoor and Mobile Radio Commun. (PIMRC), Berlin, Germany, Sept. 2005, vol. 3, pp. 1683-1687.

[15] D. N. Dao, C. Tellambura, C. Yuen, T. T. Tjhung, and Y. L. Guan, "Fourgroup decodable semi-orthogonal algebraic space-time block codes," in Proc. IEEE Wireless Commun. and Networking Conf. (WCNC), Hong Kong, China, Mar. 2007, pp. 588-593.

[16] D. N. Dao, C. Yuen, C. Tellambura, Y. L. Guan, and T. T. Tjhung,
"Four-group decodable space-time block codes," IEEE Trans. Signal Processing, vol. 56, pp. 424-430, Jan. 2008.

[17] V. Tarokh, H. Jafarkhani, and A. R. Calderbank, "Space-time codes for high data rate wireless communication: performance criterion and code construction," IEEE Trans. Inform. Theory, vol. 44, pp. 744-765, Mar. 1998.

[18] J. Yuan, Z. Chen, B. Vucetic, and W. Firmanto, "Performance and design of space-time coding in fading channels," IEEE Trans. Commun., vol. 51, pp. 1991-1996, Dec. 2003.

[19] W. Su, Z. Safar, and K. J. R. Liu, "Diversity analysis of space-time modulation over time-correlated Raleigh fading channels," IEEE Trans. Inform. Theory, vol. 50, pp. 1832-1839, Aug. 2004.

[20] D. Carlson, C. R. Johnson, D. Lay, and A. D. Porter, "Gems of exposition in elementary linear algebra," College Math. J., vol. 23, no. 4, pp. 299-303, Sept. 1992. 